SELECCIONES MATEMÁTICAS
Universidad Nacional de Trujillo
ISSN: 2411-1783 (Online)
2019; Vol.06(2): $148-155$.

\title{
Soluciones Numéricas para la Ecuación KdV Usando el Método Wavelet-Petrov-Galerkin.
}

\section{Numerical Solutions of the KdV Equation for Wavelet-Petrov-Galerkin Method.}

\author{
Julio Cesar Duarte Vidal *(iD) and Francisco Javier Reyes Bahamón t'iD
}

Received, Aug. 01, 2019

Accepted, Oct. 14, 2019

How to cite this article:

Duarte, J., Reyes, F. Soluciones Numéricas para la Ecuación KdV Usando el Método Wavelet-Petrov-Galerkin. Selecciones Matemáticas. 2019; 6(2):148-155. http://dx.doi.org/10.17268/sel .mat.2019.02.02

\begin{abstract}
Resumen
Este trabajo Contiene la solución numérica de la ecuación KdV usando el método de Petrov-Galerkin-Wavelet. Lo interesante es poder calcular las integrales Wavelets, usando Wavelets Biortogonales, las propiedades de simetría permiten que los cálculos se reduzcan ostensiblemente. Aquí aplicaremos conceptos del análisis funcional y la teoría de distribuciones inmersos en el cálculo de la derivada débil o derivada distribucional. Hasta obtener gráficamente la solución numérica y la solución analítica de esta ecuación muy usada en la parte de la tecnología de ondas y comunicaciones, como también en la reconstrucción de imágenes. Recientemente, los métodos de wavelet se aplican a la solución numérica de ecuaciones diferenciales parciales, trabajos pioneros en esta dirección son las de Beylkin, Dahmen, Jaffard y Glowinski, entre otros.
\end{abstract}

Palabras clave. Ecuación KdV, Método de Petrov-Galerkin, Wavelets Biortogonales, Ecuación diferencial parcial, Integrales Wavelets.

Abstract
This work contains the numerical solution of the KdV equation using the Petrov-Galerkin-Wavelet method. The interesting thing is to be able to calculate Wavelet integrals, using Biorthogonal Wavelets, the properties of symmetry allow the calculations to be significantly reduced. Here we will apply concepts of functional analysis and the theory of distributions immersed in the calculation of the weak derivative or distributional derivative. To obtain graphically the numerical solution and the analytical solution of this equation very used in the part of the wave and communications technology, as well as in the reconstruction of images. Recently, wavelet methods are applied to the numerical solution of partial differential equations, pioneering works in this direction are those of Beylkin, Dahmen, Jaffard and Glowinski, among others.

Keywords. KdV Equation, Method Pretov-Galerkin, Wavelets Biorthogonals, Partial Differential Equation, Integrals Wavelets.

1. Introducción. El objetivo principal de este trabajo es presentar una solución numérica de la ecuación Kortewegde de Vries (KdV)

$$
\frac{\partial u}{\partial t}+\mu u \frac{\partial u}{\partial x}+\epsilon \frac{\partial^{3} u}{\partial x^{3}}=0
$$

\footnotetext{
*Facultad de Educación, Universidad Surcolombiana, Neiva-Huila, Colombia (juli o. duarte@usco.edu . co).

${ }^{\dagger}$ Facultad de Educación, Universidad Surcolombiana, Neiva-Huila, Colombia (f jreyesb@unal . edu. co),
} 
con la condición inicial $u(x, 0)=u_{0}(x)$, donde $\mu \mathrm{y} \epsilon$ son constantes positivas, el cual usamos el método de Petrov-Galerkin-Wavelet. Esta ecuación aparece en el estudio de ondas en aguas poco profundas en la dinámica de fluidos $[9,10,15]$. La ecuación $\mathrm{KdV}$ satisface la propiedad que el término no lineal $u u_{x}$ y la dispersión $u_{x x x}$ se balancean generando equilibrio entre sí generando soluciones de onda que se propagan manteniendo la misma forma. El término Solitón fue acuñado por Zabusky y Kruskal para describir esta onda solitaria, solución de la ecuación $\mathrm{KdV}$ [2,9, 11].

Daubechies presenta un método para construir wavelets con soporte compacto y funciones de escala con regularidad arbitraria y momento cero [8]. Sin embargo, el precio de estas buenas propiedades es la ausencia de simetría y suporte amplio. Esta desventaja desaparece en el contexto de las ondas biortogonales, un concepto introducido por Cohen, Daubechies y Feauveau en [6]. En este contexto, dos funciones base no ortogonales $\psi_{j, k} \mathrm{y}$ $\psi_{j, k}^{*}$ también llamado Wavelets, son construidas con base en funciones escalas trasladadas $\psi \mathrm{y} \psi^{*}$.

A diferencia del método de Galerkin, donde se utilizan las mismas funciones base como prueba y admisible, en el método de Petrov-Galerkin, las funciones de prueba y admisibles son diferentes. En la aproximación de PetrovGalerkin usando wavelets biortogonales, la idea es tener una de las familias de funciones base como admisibles y su dual como funciones de prueba. La ventaja de este método es el preacondicionamiento y discretización de las wavelets para algoritmos adaptativos $[5,17,20]$. Por lo tanto la técnica wavelets proporcionan métodos numéricos eficientes, como alternativa a los métodos clásicos [1,7,12,13,17].

El objetivo de este trabajo es estudiar la precisión del método de Petrov-Galerkin mediante el uso de wavelets biortogonales, en la solución de la ecuación $\mathrm{KdV} u_{t}+\mu u u_{x}+\epsilon u_{x x x}=0$ con la condición inicial $u(x, 0)=u_{0}(x)$, En lugar de Bases wavelet multinivel, ampliamos las soluciones aproximadas en términos de funciones de escala $\phi_{m, k}(x)$ de un solo nivel como base para funciones admisibles, mientras que el dual $\phi_{m, k}^{*}(x)$ son las funciones de prueba.

El artículo esta organizado de la siguiente manera: después de algunos comentarios preliminares en la sección 1, en la Sección 2, damos y discutimos algunos hechos que muestran las ideas fundamentales del método. En la sección 3 discutimos los algoritmos para calcular las integrales wavelets como $a(k), b(l, k)$ y $c(k)$, también publicamos los resultados numéricos y los errores.

2. Método de Petrov-Galerkin para la ecuación KdV. El método de Petrov-Galerkin es un caso particular de un método más general, conocido como Método de residuos ponderados [10,15]. Consideremos la formulación débil de la ecuación $\mathrm{KdV}$. Sea $0 \leq \alpha \leq 3$ y $\beta=3-\alpha$, para toda función de prueba $v$, $\beta$-regular, se tiene

$$
\int_{\mathbb{R}} v \frac{\partial u}{\partial t} d x+\mu \int_{\mathbb{R}} v u \frac{\partial u}{\partial x} d x+\epsilon \int_{\mathbb{R}} v \frac{\partial^{3} u}{\partial x^{3}} d x=0
$$

Luego la formulación débil se puede expresar como

$$
\left(\frac{\partial u}{\partial t}, v\right)+\mu\left(u \frac{\partial u}{\partial x}, v\right)+\epsilon(-1)^{\beta}\left(\frac{\partial^{\alpha} u}{\partial x^{\alpha}}, \frac{d^{\beta} v}{d x^{\beta}}\right)=0
$$

donde $(\cdot, \cdot)$ es el producto interno en $L_{2}(\mathbb{R})$. Recordemos que una función $g$ es $r$-regular, si $x^{n} g^{(s)}(x) \in$ $L_{2}(\mathbb{R})$ para todo $s$ tal que $0 \leq s \leq r$ y para todo $n \in \mathbb{N}$. O también, si existe una constante $M_{s, n}>0$ tal que $\left|g^{(s)}(x)\right| \leq M_{s, n}(1+|x|)^{-n}$, para cada $x \in \mathbb{R}$, para todo índice $s$ tal que $0 \leq s \leq r$ y para todo $n \in \mathbb{N}(16)$.

Aplicando el método de Petrov-Galerkin, tomando como funciones admisibles $\phi_{h, k}(x)=h^{-1 / 2} \phi\left(h^{-1} x-\right.$ $k), \quad k \in \mathbb{Z}$, donde $\phi$ es una función de valor real, $r$-regular, $r \geq 1$, y $h>0$ es el paso de discretización. Los espacios de aproximación $V_{h} \subset L_{2}(\mathbb{R})$ son generados por $\left\{\phi_{h, k}(x), k \in \mathbb{Z}\right\}$, y la solución exacta de la ecuación $\mathrm{KdV}(1.1)$, se aproxima por la expresión $u_{h}(x, t)=\sum_{k} U_{k}(t) \phi_{h, k}(x)$. Similarmente, las funciones de prueba se toman de la forma $\phi_{h, k}^{*}(x)$, definidas en términos de una función $r^{*}-$ regular dual $\phi^{*}$, con $r+r^{*} \geq 3$.

En la formulación débil (2.1) escogemos $\alpha \leq r$ tal que $\beta \leq r^{*}$. Si reemplazamos $u$ por la solución $u_{h}(x, t)$ y 
$v$ por cada función de prueba $\phi_{h, l}^{*}(x)$, entonces

$$
\begin{aligned}
& \left(\frac{\partial u}{\partial t}, v\right)+\mu\left(u \frac{\partial u}{\partial x}, v\right)+\epsilon(-1)^{\beta}\left(\frac{\partial^{\alpha} u}{\partial x^{\alpha}}, \frac{d^{\beta} v}{d x^{\beta}}\right)= \\
& \int_{\mathbb{R}} \frac{\partial}{\partial t}\left(\sum_{k} \phi_{h, k}(x) U_{k}(t)\right) \phi_{h, l}^{*}(x) d x+ \\
& +\mu \int_{\mathbb{R}}\left(\sum_{s} U_{s}(t) \phi_{h s}(x)\right)\left(\frac{\partial}{\partial x} \sum_{k} U_{k}(t) \phi_{h k}(x)\right)\left(\phi_{h, l}^{*}(x)\right) d x+ \\
& +(-1)^{\beta} \epsilon \int_{\mathbb{R}}\left(\frac{\partial^{\alpha}}{\partial x^{\alpha}} \sum_{k} U_{k}(t) \phi_{h k}(x)\right)\left(\frac{d^{\beta}}{d x^{\beta}} \phi_{h, l}^{*}(x)\right) d x \\
& =h^{-1} \sum_{k} \int_{\mathbb{R}} \phi\left(h^{-1} x-k\right) \phi^{*}\left(h^{-1} x-l\right) \frac{d U_{k}(t)}{d t} d x+ \\
& +\mu h^{-3 / 2} \sum_{s} \sum_{k} \int_{\mathbb{R}} \phi\left(h^{-1} x-s\right) \phi^{*}\left(h^{-1} x-l\right) \frac{d}{d x} \phi\left(h^{-1} x-k\right) U_{s}(t) U_{k}(t) d x+ \\
& +h^{-1} \epsilon(-1)^{\beta} \sum_{k} \int_{\mathbb{R}} \frac{d^{\alpha}}{d x^{\alpha}} \phi\left(h^{-1} x-k\right) \frac{d^{\beta}}{d x^{\beta}} \phi^{*}\left(h^{-1} x-l\right) U_{k}(t) d x=0 .
\end{aligned}
$$

Si ponemos $U_{k}(t)=U_{k}$ y hacemos el cambio de variable $y=h^{-1} x-k$, la expresión anterior la podemos escribir como

$$
\sum_{k} a(k) \frac{d U_{k}}{d t}+\mu h^{-3 / 2} \sum_{s} \sum_{k} b(l, k) U_{s} U_{k}+h^{-3} \epsilon \sum_{k} c(k) U_{k}=0
$$

donde

$$
\begin{gathered}
a(k)=\int_{\mathbb{R}} \phi(y) \phi^{*}(y-k) d y \\
b(l, k)=\int_{\mathbb{R}} \frac{d \phi(y)}{d y} \phi(y-l) \phi^{*}(y-k) d y \\
c(k)=(-1)^{\beta} \int_{\mathbb{R}} \frac{d^{\alpha} \phi(y)}{d y^{\alpha}} \frac{d^{\beta} \phi^{*}(y-k)}{d y^{\beta}} d y .
\end{gathered}
$$

Los coeficientes $U_{k}$ se determinan del siguiente sistema de ecuaciones diferenciales ordinarias

$$
\sum_{k} a(l-k) \frac{d}{d t} U_{k}+\mu h^{-3 / 2} \sum_{s} \sum_{k} b(s-k, l-k) U_{s} U_{k}+h^{-3} \epsilon \sum_{k} c(l-k) U_{k}=0 .
$$

La forma matricial de esta última ecuación es

$$
\frac{d}{d t} L U+\mu U^{T} M U+\epsilon N U=0
$$

donde $U=\left(U_{k}\right), \quad L(l, k)=a(l-k), \quad M(l, k, s)=h^{-3 / 2} b(l-k, l-s) \quad N(l, k)=h^{-3} c(l-k)$. Las condiciones iniciales $U_{k}(0), k \in \mathbb{Z}$, son los coeficientes de $u_{h}(x, 0)=R_{h} u_{0} \in V_{h}$, donde $R_{h}$ es algún esquema de aproximación inicial que se precisará más adelante.

Con un paso de tiempo $\Delta t=t_{n+1}-t_{n}$ y aplicando la regla del trapezoidal se tiene $\frac{d U}{d t}=\frac{U^{n+1}-U^{n}}{\Delta t}$, donde $U^{n}=U(n \Delta t)$ para $n \geq 0$, luego la ecuación (2.3) queda

$$
L\left[\frac{U^{n+1}-U^{n}}{\Delta t}\right]+\mu U^{T} M U+\epsilon N U=0 .
$$

Haciendo $G(U)=\mu U^{T} M U+\epsilon N U$ se tiene

$$
L\left(U^{n+1}-U^{n}\right)+\frac{G\left(U^{n+1}\right)+G\left(U^{n}\right)}{2} \Delta t=0,
$$

esta ecuación se resuelve por el método de iteración de Newton. 
3. Algoritmos para calcular $a(k), b(l, k)$ y $c(k)$. Para calcular los coeficientes $a(k), b(l, k)$ y $c(k)$ recurriremos a resolver (parcialmente) el problema de valor de frontera periódico para la ecuación diferencial con coeficientes constantes

$$
\begin{gathered}
\sum_{s=0}^{d} a_{s} D^{s} u \equiv \mathcal{P} u=g \\
u \quad \text { y } \quad g \quad 1-\text { periódicas en el espacio }
\end{gathered}
$$

donde $D=\frac{d}{d x}$. El método de Galerkin descrito en esta sección se basa en el marco biortogonal $\left\{V_{j}, V_{j}^{*}\right\}$, asociado a las funciones de escala spline biortogonal $\phi=\phi_{N} \mathrm{y} \phi^{*}=\phi_{N, N^{*}}$, con el correspondiente par conjugado $\left\{\tilde{\phi}, \widetilde{\phi^{*}}\right\}$, donde

$$
\tilde{\phi}(x)= \begin{cases}\phi_{N-1}(x) & \text { si N es impar } \\ \phi_{N-1}(x+1) & \text { si N es par. }\end{cases}
$$

$\mathrm{y}$

$$
\widetilde{\phi^{*}}(x)= \begin{cases}\phi_{N-1, N^{*}+1}(x) & \text { si } \mathrm{N} \text { es impar } \\ \phi_{N-1, N^{*}+1}(x+1) & \text { si } \mathrm{N} \text { es par. }\end{cases}
$$

Una de las familias de funciones base se utilizará como funciones de ensayo o admisibles, digamos $\phi_{j, k}(x)$, y su familia dual $\phi_{j, k}^{*}(x)$ como funciones de prueba. Esto significa que la solución aproximada para el problema de valor de frontera dado es

$$
u_{j}(x)=\sum_{k \in \mathbb{Z}} U_{j}(k) \phi_{j, k}(x)
$$

donde $U_{j}(k)=U_{j}\left(k+2^{j}\right)$ son los coeficientes periódicos que se deben determinar a través de la siguiente relación

$$
\int_{\mathbb{R}}\left(\mathcal{P} u_{j}-g\right) \phi_{j, l}^{*}(y) d y=0 \quad \text { para cada } \quad l \in \mathbb{Z} .
$$

Siempre que las funciones base tengan la suficiente regularidad para que esta integral tenga sentido. La ecuación (3.1) se puede expresar como un sistema de ecuaciones diferenciales ordinarias

$$
\sum_{k \in \mathbb{Z}} \sum_{s=0}^{d} 2^{s j} \Gamma(k-l) U_{j}(k)=G_{j}(l) \quad \text { para cada } l \in \mathbb{Z},
$$

donde $G_{j}(k)=\left\langle g, \psi_{j, k}^{*}\right\rangle \mathrm{y}$

$$
\Gamma_{s}(m)=\Gamma_{s, N, N^{*}}(m)=(-1)^{p} \int_{\mathbb{R}} D^{s-p} \phi(y) D^{p} \phi^{*}(y+m) d y,
$$

los indices $0 \leq p \leq s$ se introducen aquí con el fin de controlar la posible falta de regularidad de las funciones base [13]. Para ilustrar el cálculo de los coeficientes utilizando la fórmula (3.3), lo haremos para la función hat, con $0 \leq s \leq 3$,

$$
\phi(x)=\phi_{2}(x)=\left\{\begin{array}{l}
1+x \text { si }-1 \leq x<0 \\
1-x \text { si } 0 \leq x \leq 1 \\
0 \text { en otro caso }
\end{array}\right.
$$

y sus duales $\phi^{*}=\phi_{2, N^{*}}$ con $N^{*} \geq 4$, que tienen soporte sobre los intervalos $\left[-N^{*}, N^{*}\right]$.

4. Simulación Matemática. De la relación de biortogonalidad se tiene

$$
\Gamma_{0}(m)=a(k)=\int_{\mathbb{R}} \phi(y) \phi^{*}(y) d y=\delta_{k, 0} .
$$

Si derivamos la función hat obtenemos

$$
\frac{d \phi_{2}(x)}{d x}=\left\{\begin{array}{rr}
1 & -1<x<0 \\
-1 & 0<x<1 \\
0 & \text { en otro caso }
\end{array}\right.
$$


entonces

$$
d(-m)=\Gamma_{1}(m)=\int_{\mathbb{R}} \frac{d \phi(y)}{d y} \phi^{*}(y+m) d y=\int_{-1}^{0} \phi^{*}(y+m) d y-\int_{0}^{1} \phi^{*}(y+m) d y
$$

para $s=1, p=0$ y $N=2$

$$
\widetilde{\phi^{*}}(x)=\tilde{\phi}_{2, N^{*}}(x)=\phi_{1, N^{*}+1}(x+1)=\int_{x}^{x+1} \phi_{2, N^{*}}(y) d y
$$

aplicando estos cálculos obtenemos

$$
\begin{aligned}
\Gamma_{1}(m) & =\int_{-1}^{0} \phi_{2, N^{*}}(y+m) d y-\int_{0}^{1} \phi_{2, N^{*}}(y+m) d y \\
& =\int_{-1+m}^{m} \phi_{2, N^{*}}(z) d z-\int_{m}^{1+m} \phi_{2, N^{*}}(z) d z \\
& =\phi_{1, N^{*}+1}(m)-\phi_{1, N^{*}+1}(m+1) .
\end{aligned}
$$

La segunda derivada de la función hat en el sentido de las distribuciones es

$$
\begin{aligned}
\left\langle\phi_{2}^{\prime \prime}(x), \varphi\right\rangle & =(-1)^{2}\left\langle\phi_{2}(x), \varphi^{\prime \prime}(x)\right\rangle=\int_{-\infty}^{\infty} \phi_{2}(x) \varphi^{\prime \prime}(x) d x \\
& =\left.(x+1) \varphi^{\prime}(x)\right|_{-1} ^{0}+\left.(1-x) \varphi^{\prime}(x)\right|_{0} ^{1} \\
& =\varphi^{\prime}(0)-\left.\varphi(x)\right|_{-1} ^{0}-\varphi^{\prime}(0)+\left.\varphi(x)\right|_{0} ^{1} \\
& =-\varphi(0)+\varphi(-1)+\varphi(1)-\varphi(0) \\
& =\delta(x+1)-2 \delta(x)+\delta(x-1)
\end{aligned}
$$

con $s=2$ y $p=1$ tenemos

$$
\begin{aligned}
\Gamma_{2} & =(-1) \int_{\mathbb{R}} \phi^{\prime}(y) \phi^{*^{\prime}}(y+m) d y=-\int_{-1}^{0} \phi^{*^{\prime}}(y+m) d y+\int_{0}^{1} \phi^{*^{\prime}}(y+m) d y \\
& =-\phi^{*}(m)+\phi^{*}(-1+m)+\phi^{*}(1+m)-\phi^{*}(m) \\
& =\phi_{2, N^{*}}(m-1)-2 \phi_{2, N^{*}}(m)+\phi_{2, N^{*}}(1+m)
\end{aligned}
$$

Nótese que $\phi_{2, N^{*}}(x)=\int_{x}^{x+1} \phi_{3, N^{*}-1}(y) d y, \mathrm{y} \operatorname{con} N=3$

$$
\widetilde{\phi^{*}}(x)=\phi_{2, N^{*}+1}(x)=\int_{x}^{x+1} \phi^{*}(y) d y=\int_{x}^{x+1} \phi_{3, N^{*}}(y) d y .
$$

Para el cálculo de la integral $c(k)$ tomamos los valores $s=3$ y $p=1$, también es válido para valores de $\alpha=2$ y $\beta=1$

$$
\begin{aligned}
\Gamma_{3}(m) & =(-1) \int_{\mathbb{R}} \phi^{\prime \prime}(y){\phi^{*^{\prime}}(y+m) d y} \\
& =-\int_{\mathbb{R}}[\delta(y+1)-2 \delta(y)+\delta(y-1)]\left[\phi^{*^{\prime}}(y+m)\right] d y \\
& =\int_{\mathbb{R}}[\delta(y+1)-2 \delta(y)+\delta(y-1)]\left[\phi_{3, N^{*}-1}(y+m+1)-\phi_{3, N^{*}-1}(y+m)\right] d y \\
& =-\int_{\mathbb{R}}\left[\delta(y+1) \phi_{3, N^{*}-1}(y+m+1)-2 \delta(y) \phi_{3, N^{*}-1}(y+m+1)+\right. \\
& +\delta(y-1) \phi_{3, N^{*}-1}(y+k+1)-\delta(y+1) \phi_{3, N^{*}-1}(y+k)+ \\
& \left.+2 \delta(y) \phi_{3, N^{*}-1}(y+m)-\delta(y-1) \phi_{3, N^{*}-1}(y+m)\right] d y \\
& =-\left[\phi_{3, N^{*}-1}(m)-2 \phi_{3, N^{*}-1}(m+1)+\phi_{3, N^{*}-1}(m+2)-\phi_{3, N^{*}-1}(m-1)+\right. \\
& \left.+2 \phi_{3, N^{*}-1}(m)-\phi_{3, N^{*}-1}(m+1)\right] \\
& =\phi_{3, N^{*}-1}(m-1)+3 \phi_{3, N^{*}-1}(m+1)-3 \phi_{3, N^{*}-1}(m)-\phi_{3, N^{*}-1}(m+2)
\end{aligned}
$$

luego

$$
c(k)=\Gamma_{3}(k)=\phi_{3, N^{*}-1}(k-1)+3 \phi_{3, N^{*}-1}(k+1)-3 \phi_{3, N^{*}-1}(k)-\phi_{3, N^{*}-1}(k+2) .
$$


Si bien, para el cálculo de la integral $b(l, k)$ no seguimos el mismo procedimiento, si utilizamos algunos resultados obtenidos anteriormente

$$
\begin{aligned}
b(l, k) & =\int_{-\infty}^{+\infty} \frac{d \phi_{2}(x)}{d x} \phi_{2}(x-l) \phi^{*}(x-k) d x \\
& =\int_{-1}^{0} \phi_{2}(x-l) \phi_{2, N^{*}}(x-k) d x-\int_{0}^{1} \phi_{2}(x-l) \phi_{2, N^{*}}(x-k) d x \\
& =\int_{-1-k}^{-k} \phi_{2}(y+k-l) \phi_{2, N^{*}}(y) d y-\int_{-k}^{1-k} \phi_{2}(y+k-l) \phi_{2, N^{*}}(y) d y \\
& =\int_{l-1-k}^{l-k}(1+y+k-l) \phi_{2, N^{*}}(y) d y-\int_{l-k}^{1+l-k}(1-y-k+l) \phi_{2, N^{*}}(y) d y \\
& =(1+k-l) \int_{-1-k+l}^{-k+l} \phi_{2, N^{*}}(y) d y+\int_{-1-k+l}^{-k+l} y \phi_{2, N^{*}}(y) d y+ \\
& +\int_{-k+l}^{-1-k+l} y \phi_{2, N^{*}}(y) d y-(1-k+l) \int_{-k+l}^{-1-k+l} \phi_{2, N^{*}}(y) d y
\end{aligned}
$$

por lo tanto,

$$
b(l, k)=I(-1-k+l)+I(-k+l)+(1+k-l) \phi_{1, N^{*}+1}(l-k)-(1-k+l) \phi_{1, N^{*}+1}(1-k+l),
$$

donde

$$
I(k)=\int_{k}^{k+1} y \phi_{2, N^{*}}(y) d y .
$$

Para $l=0$;

$$
b(0, k)=I(-1-k)+I(-k)+(1+k) \phi_{1, N^{*}+1}(-k)-(1-k) \phi_{1, N^{*}+1}(1-k) .
$$

Para $l=-1$

$$
b(-1, k)=I(-2-k)+I(-1-k)+(2+k) \phi_{1, N^{*}+1}(-1-k)+k \phi_{1, N^{*}+1}(-k) .
$$

Para $l=1$,

$$
b(1, k)=I(-k)+I(1-k)+k \phi_{1, N^{*}+1}(1-k)-(2-k) \phi_{1, N^{*}+1}(2-k) .
$$

Nótese que $I(1-k)+(k-2) \phi_{1, N^{*}+1}(2-k)=0$. Luego

$$
b(1, k)=I(-k)+k \phi_{1, N^{*}+1}(1-k) .
$$

De igual forma se tiene para $l=-1$ que

$$
b(-1, k)=I(-1-k)+k \phi_{1, N^{*}+1}(-k) .
$$

Por simetría de $\phi_{2, N^{*}}(y)$ alrededor de $x=0$ se tiene

$$
\begin{aligned}
I(k) & =\int_{k}^{k+1} \phi_{2, N^{*}}(y) d y=-\int_{k+1}^{k} y \phi_{2, N^{*}}(y) d y=-\int_{-k-1}^{-k} y \phi_{2, N^{*}}(y) d y \\
& =-I(-k-1)
\end{aligned}
$$

y la simetría de $\phi_{1, N^{*}-1}(x)$ alrededor de $x=\frac{1}{2}$, implica

$$
b(-1, k)=-b(1,-k) \quad \text { y } \quad b(0, k)=-b(0,-k) .
$$

De la condición de biortogonalidad aplicada a $\phi=\phi_{2}$ y $\phi^{*}=\phi_{2, N *}$ se obtiene la siguiente relación

$$
\int_{-\infty}^{+\infty} \phi(y) \phi^{*}(y-k) d y=\delta_{k, 0}
$$


de esta manera se obtiene

$$
\begin{aligned}
\int_{-\infty}^{+\infty} \phi_{2}(y) \phi_{2, N^{*}}(y-k) d y & =\int_{-1-k}^{-k}(1+z+k) \phi_{2, N^{*}}(z) d z+ \\
& +\int_{-k}^{1-k}(1-z-k) \phi_{2, N^{*}}(z) d z \\
& =\int_{-1-k}^{-k} z \phi_{2, N^{*}}(z) d z-\int_{-k}^{1-k} z \phi_{2, N^{*}}(z) d z+ \\
& +(1+k) \int_{-1-k}^{-k} \phi_{2, N^{*}}(z) d z+ \\
& +(1-k) \int_{-k}^{1-k} \phi_{2, N^{*}}(z) d z=\delta_{k, 0} .
\end{aligned}
$$

Luego

$$
I(-1-k)-I(-k)+(k+1) \phi_{1, N^{*}+1}(-k)+(1-k) \phi_{1, N^{*}+1}(1-k)=\delta_{k, 0}
$$

esta fórmula se puede usar para simplificar la expresión $b(0, k)$ dando

$$
b(0, k)=\delta_{0, k}+2 I(-k)-2(1-k) \phi_{1, N^{*}+1}(k) .
$$

El algoritmo para el cálculo de $I(k)$ es el siguiente:

- $I(0)=\phi_{1, N^{*}+1}(0)-\frac{1}{2}$

- Para $k=1,2, \ldots, N^{*}-1$

$-I(-k)=-I(k-1)$

$-I(k)=-I(-k)+(k+1) \phi_{1, N^{*}+1}(-k)+(1-k) \phi_{1, N^{*}+1}(k)$

- $I\left(-N^{*}\right)=-I\left(N^{*}-1\right)$.

Para llevar a cabo los cálculos es necesario tener en cuenta los valores de $\phi_{1, N^{*}+1}$ y $\phi_{3, N^{*}+1}$ en los enteros. En general, el orden para calcular los valores de la función escala de soporte compacto en los enteros, puede usarse el algoritmo de cascada, que empieza con la ecuación de dilatación y soluciona el problema de valores propios para la matriz cuyas entradas son $h(2 l-k)$.

\begin{tabular}{|c|c|}
\hline Tiempo & Error \\
\hline 0 & $2,53 * \mathrm{e}-18$ \\
\hline 0,1 & 0,04114137 \\
\hline 0,2 & 0,04298284 \\
\hline 0,3 & 0,05190608 \\
\hline 0,4 & 0,06032352 \\
\hline 0,5 & 0,07034124 \\
\hline 0,6 & 0,08141202 \\
\hline 0,7 & 0,09275702 \\
\hline 0,8 & 0,10438704 \\
\hline 0,9 & 0,11619728 \\
\hline \multicolumn{2}{|c|}{ CUADRO 4.1} \\
\hline
\end{tabular}

Error del método con $h=2^{-5}$

\section{Conclusions.}

1. Las propiedades de las Wavelets Biortogonales hacen que los cálculos computacionales se reduzcan sustancialmente y las aproximaciones sean muy buenas.

2. El Método de Petrov-Galerkin-Wavelets con el transcurrir del tiempo se adapta muy bien, manteniendo la forma de onda y manteniendo una muy buena convergencia de la solución analítica con la solución numérica, aunque al transcurrir el tiempo, el error aumenta muy poco.

3. Este método transforma una ecuación diferencial parcial en un sistema de ecuaciones lineales, los elementos de la matriz del sistema son integrales Wavelets Biortogonales y se hizo un estudio detallado donde se muestra propiedades de simetría y otra clase de cálculos que hace que el sistema sea fácil de resolver, así muestra que tanto $a(k)$, como $b(l, k)$ son fáciles de calcular y $c(k)$ es un poco más complejo. 


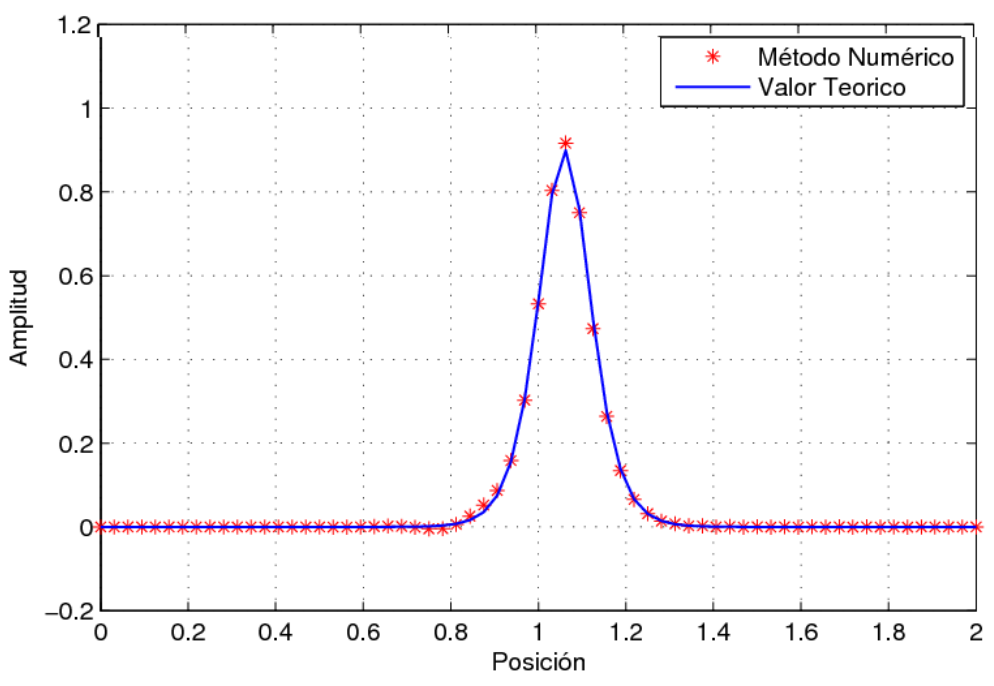

FIGURA 4.1. Comparación entre el método y la solución exacta

6. Acknowledgements. We gratefully thank Dr. José Curto and Dr. John Frain for providing some of the data sets used in this work. The authors also wish to thank J. P. Nolan who kindly gave his Rstable package for computing stable densities in this work. The stable package is available at urlhttp://RobustAnalysis.com. S.R.C. Lopes research was partially supported by CNPq-Brazil and by INCT em Matemática.

\section{ORCID and License}

Julio Cesar Duarte Vidal https://orcid.org/0000-0001-9407-5721,

Franciso Javier Reyes Bahamón https://orcid.org/0000-0002-1843-2894.

This work is licensed under the Creative Commons Attribution-NoComercial-ShareAlike 4.0.

Referencias

[1] Beylkin, G., Keiser, J. M. On the Adaptive Numerical Solution of Nonlinear Partial Differential Equations in Wavelet bases, Journal of Computational Physics. 1997; 132:233-259.

[2] Biswas, A., Konar, S. Soliton perturbation theory for the compound KdV equation. International Journal of Theoretical Physics. 2007; 46(2):237 - 243.

[3] Bleistein, N., Handelsman, R. Asymptotic Expansions of Integrals. Dover Publications, Inc. New York; 1986.

[4] Canivar, A. sari, M. and Dag, I., A Taylor - Galerkin finite element method for the KdV equation using cubic B-Splines. Physica B. 2010; 405:3376-3383.

[5] Chiavassa, G., Liandrat, J. A fully adaptive wavelet algorithm for parabolic partial differential equations, Applied Numerical Mathematics. 2001; 36:333-358.

[6] Cohen, A., Daubechies, I., and Feauveau, J. C. Biorthogonal basis of compactly supported wavelets, Comm. Pure Appl. Math. 1992; 45:485-560.

[7] Dahmen, W. Wavelet methods for PDEs-some recent developments, Journal of Computational and Applied Mathematics. 2001; 128:133185.

[8] Daubechies, I. Orthonormal bases of compactly supported wavelets, Comm. Pure Appl. Math. 1988; 41:909-996.

[9] Debnath, L. Nonlinear Partial Differential Equations: for Scientists and Engineers. Second Edition, Birkhäuser, Boston; 2005.

[10] Donea, J. Huerta, A. Finite Element Methods for Flow problems. John Wiley and Sons, Ltd. England, 2003.

[11] Drazin, P. G., Johnson, R. S. Solitons: An Introduction. Second Edition, Cambridge University Press, New York, 1989.

[12] Glowinski, R., Lawton, W., Ravachol, M., and Tenenbaum, E. Wavelet solution of linear and non-linear elliptic, parabolic and hyperbolic problems in one dimension, pp 55-120 in Computing Methods in Applied Science and Engineering, R. Glowinski and A. Lichnewski, (eds.), SIAM, Philadelphia, PA, 1990.

[13] Gomes, S., Cortina, E. Fourier Analysis of Petrov-Galerkin Methods Based on Biorthogonal Multiresolution Analysis, pp 119-140 in Wavelets Theory and Harmonic Analysis in Applied Sciences, C. E. D’Attellis, E. M. Fernández-Berdaguer (eds.), Birkhäuser, Boston, 1997.

[14] Lemarié, F. G. Functions a support compact dans les analyses multiresolutions, Revista matemática Iberoamericana, 1991; 7(2):157 182.

[15] Lewis, R. W., Nithiarasu, P., Seetharamu, K. N. Fundamentals of the finite element method for Heat and Fluid Flow. John Wiley and Sons, Inc. New York, 2004.

[16] Meyer, Y. Ondelettes et opérateurs, I: Ondelettes. Herman, Paris, 1990.

[17] Rathish, B. V., Mehra, M. Time - accurate solutions of Korteweg-de Vries equation using wavelet Galerkin method. Applied Mathematics and Computation. 2005; 162:447 - 460.

[18] Shoombie, S. W. Spline Petrov - Galerkin methods for the numerical solution of the Korteweg-de Vries equation. IMA Journal of Numerical Analysis, 1982; 2:95-109.

[19] Shen, X. A Galerkin-wavelet method for a singular convolution equation on the real line, J. Int. Equa. Appl. 2000; 12:157-176.

[20] Urban, K. Wavelet Methods for Elliptic Partial Differential Equations. Oxford University Press Inc., New York, 2009. 